\title{
INFLUENCE OF ELECTRIC FIELD ON THERMO-CHEMICAL CONVERSION OF MIXTURES OF STRAW PELLETS WITH COAL
}

\author{
Harijs Kalis ${ }^{1}$, Maksims Marinaki ${ }^{1}$, Uldis Strautins ${ }^{1}$, Inesa Barmina ${ }^{2}$ \\ ${ }^{1}$ Institute of Mathematics and Computer Science, University of Latvia, Latvia; \\ ${ }^{2}$ Institute of Physics, University of Latvia, Latvia \\ kalis@lanet.lv, maksims.marinaki@lu.lv, uldis.strautins@lu.lv,mzfi@sal.lv
}

\begin{abstract}
The recent research is focused on experimental study and mathematical modelling of the development of combustion dynamics at thermo-chemical conversion of biomass mixtures (straw pellets with crashed coal) with the aim to better understand the effect of electric field on the formation of the main gasification/ combustion characteristics when co-combusting straw with crashed coal. The experimental study and numerical modelling of the electric field effects on the combustion dynamics, when co-combusting straw pellets with coal, were carried out to ensure wider use of straw as a fuel for energy production providing the electrodynamic process control. The mathematical model considers the electric field influence on the combustion characteristics using the approximation of $2 \mathrm{D}$ axially symmetric compressible swirling flow and chemical reactions with account of the development of $\mathrm{A} \rightarrow \mathrm{B} \leftrightarrow \mathrm{C}$ kinetics ( $\mathrm{A}$ - reactant, $\mathrm{B}$ - intermediate product, $\mathrm{C}$ - final product) downstream the cylindrical combustor.
\end{abstract}

Keywords: biomass pellets, electric field, chemical reactions, mathematical model.

\section{Introduction}

The electric field effect on diffusion and premixed flames attract attention as a tool, which allows to control the flame shape, structure and the main flame characteristics, such as the flow velocity, flame temperature, composition, equivalence ratio and products composition [1-6]. There are different mechanisms of the electric field effect on the main flame characteristics. First, at high current density, the electric field effect on the flame can be related to the flame heating, with an additional heat input into the flame (plasma support of combustion). Next, the electric body forceinduces an ion drift motion in the field direction.Inelastic collisions between the flame ions and the neutral flame species can then cause the ion wind effect promoting the interrelated processes of field-enhanced heat and mass transfer in the field direction. This can cause variations of the thermal decomposition of solid fuels and the combustion of volatiles. In addition, the electric field-induced inelastic collisions between the electrons and the flame species can cause variations of the rate of reactions. Finally, the flame dynamics can be controlled using the Lorentz force, when the electric field-induced current in the flame reaction zone creates a field-induced magnetic field with axial and radial components of the electromagnetic force, which influence the evolution of flow dynamics and flow vorticity [7]. Thus, multiple factors can influence the development of combustion dynamics, when the electric field is applied to the flame. In order to obtain predictable and controllable field effect on the main flame characteristics, one can vary such parameters as the electric field polarity, bias voltage and current density between the electrodes.More precise and systematic studies of the electric field effect on the combustion dynamics for different types of flames (diffusion, swirling, etc.) and fuels (gaseous, solid and their mixtures) are required. With this account, the present paper is focused on the complex experimental study and mathematical modelling of the electric field effect on the development of swirling flame combustion dynamics at co-combustion/co-gasification of straw with a fossil fuel (coal) with the aim to assess the main factors, which affect the main gasification and combustion characteristics, if the electric field is applied to the flame base, to obtain controllable electric field effects on the combustion dynamics for more effective use of straw as a fuel for energy production. For a more detailed analysis of the processes developing at the co-combustion of straw with crashed coal, the electric field effect on the gasification/combustion characteristics at thermo-chemical conversion of the mixtures of straw pellets with crashed coal is studied, when the field is applied to the swirling flame base, with the aim to analyze the action of the electric field on the thermal decomposition of mixtures, on the ignition and combustion of volatiles. The electric field effects on the volatile combustion under predefined inlet conditions in the combustor are mathematically modelled and numerically simulated using an approximation of two dominant second-order chemical reactions developing downstream the combustor: $\mathrm{H}_{2}+\mathrm{OH} \rightarrow \mathrm{H}_{2} \mathrm{O}+\mathrm{H}$; $\mathrm{CO}+\mathrm{OH} \rightarrow \mathrm{CO}_{2}+\mathrm{H}$ [8], 
along with the estimation of electrodynamic effects due to the Lorentz force action on the combustion process in a cylindrical pipe.

\section{Mathematical modelling and numerical simulation}

A numerical simulation ofa2D axially symmetric, compressible, swirling flow (velocities $u_{r}, u_{z}$, $\left.u_{\varphi}\right)$ with density $\rho$ ' was carried out, when the volatiles $\left(\mathrm{CO}, \mathrm{H}_{2}\right)$ combustion develops downstream the cylindrical pipe (combustor) with the radius $r_{0}=0.05 \mathrm{~m}$ and length $z=z_{0}=0.1 \mathrm{~m}$. The primary air and the axial flow of volatiles, entering the combustor, were supplied at $r^{\prime} \in\left[0, r_{1}{ }_{1}\right]$ with the axial velocity $U_{0}=0.1 \mathrm{~m} \cdot \mathrm{s}^{-1}$, density $\rho_{0}=1 \mathrm{~kg} \cdot \mathrm{m}^{-3}$; the inlet temperature was $T_{0}=300 \mathrm{~K}$ and the mass fraction of reactants was $\mathrm{C}_{1}=1$. The secondary swirling air was supplied at $r^{\prime} \in\left[r_{1}{ }_{1}, r_{0}{ }_{0}\right]$ with the azimuthal velocity $V_{0}>U_{0}$ (the swirl number $S=V_{0} / U_{0}=3$ ). The computational domain used for the mathematical modelling of the electric field effect on the combustion characteristics and the experimental setup composed of a biomass gasifier and a combustor [5] are presented in Figs. 1-a, b.

a)

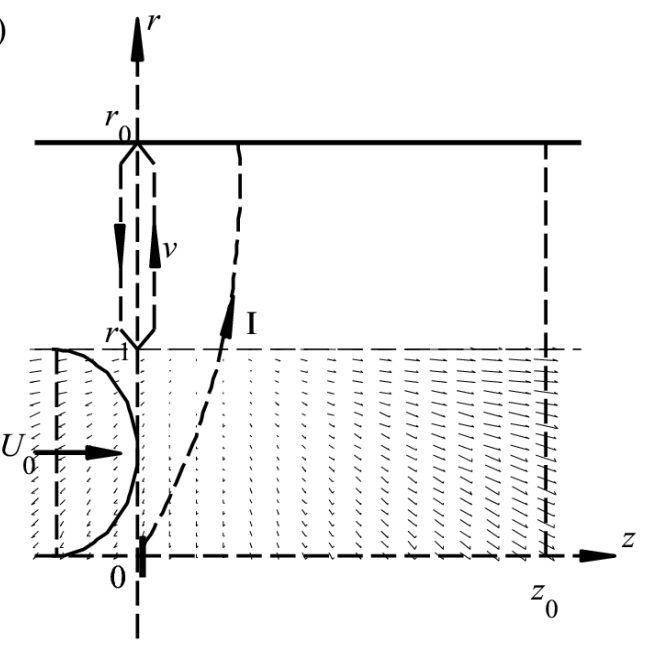

b)

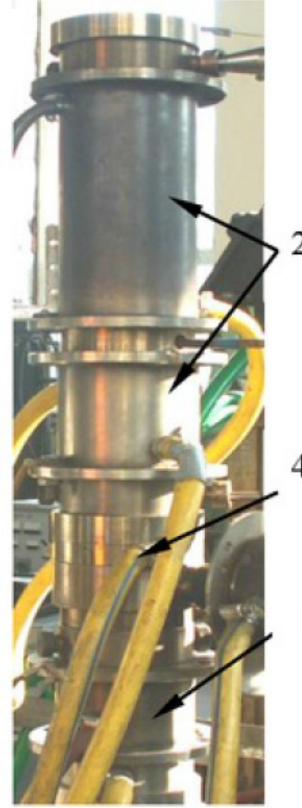

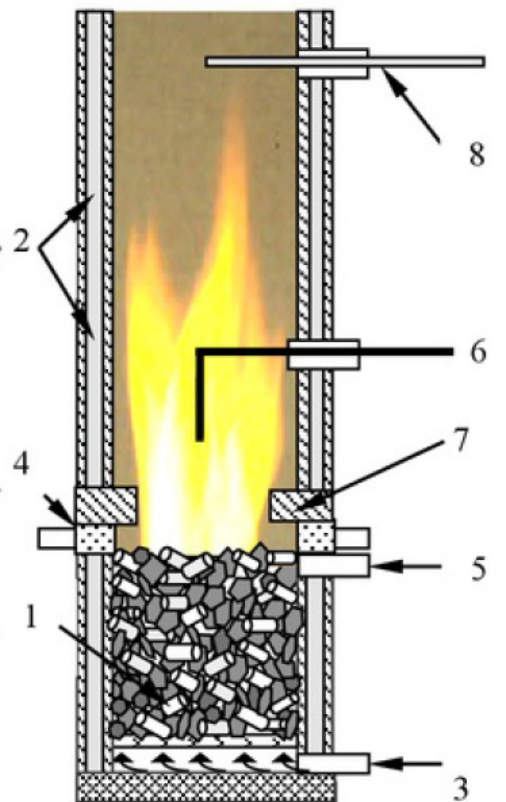

Fig. 1. Digital image of computational domain (a) and schematic of experimental device (b): 1 - gasifier with a biomass mixture; 2 - water-cooled sections of the combustor; 3 - primary air supply; 4 - secondary air supply; 5 - propane flame injection; 6 - positively biased electrode; 7 - annular inlet nozzle of the combustor; 8 - diagnostic section

The methodology of the experimental study of the electric field effect on the gasification/combustion characteristics at co-gasification/co-combustion of straw with crashed coal is described in [5].

The mathematical model is described by four Euler and three reaction-diffusion and azimuthally induced magnetic field (component $B_{\varphi}$ ) dimensionless equations in the cylindrical coordinates $(r, z)$ at time $t$ :

$$
\left\{\begin{array}{l}
\frac{\partial \rho}{\partial t}+M(\rho)+\rho\left(\frac{1}{r} \frac{\partial(r u)}{\partial r}+\frac{\partial w}{\partial x}\right)=0 \\
\frac{\partial u}{\partial t}+M(u)-S \frac{v^{2}}{r^{3}}=\frac{-1}{\rho} \frac{\partial p}{\partial r}+\operatorname{Re}^{-1}\left(\Delta u-\frac{u}{r^{2}}\right)+\frac{1}{\rho} P_{e} F_{r}, \\
\frac{\partial w}{\partial t}+M(w)=\frac{1}{\rho} \frac{\partial \rho}{x t}+\operatorname{Re}^{-1} \Delta w+\frac{1}{\rho} P_{e} F_{r}, \\
\frac{\partial v}{\partial t}+M(v)=\operatorname{Re}^{-1} \Delta_{*},
\end{array}\right.
$$




$$
\left\{\begin{array}{l}
\frac{\partial T}{\partial t}+M(T)=P_{1} \frac{1}{\rho} \Delta T+q_{1} A_{1} C_{1} \exp \left(\frac{-\delta_{1}}{T}\right)+q_{2}\left(A_{2} C_{2} \exp \left(\frac{-\delta_{2}}{T}\right)-A_{3} C_{3} \exp \left(\frac{-\delta_{3}}{T}\right)\right), \\
\frac{\partial C_{1}}{\partial t}+M\left(C_{1}\right)=P_{2} \Delta C_{1}-A_{1} C_{1} \exp \left(\frac{-\delta_{1}}{T}\right), \\
\frac{\partial C_{2}}{\partial t}+M\left(C_{2}\right)=P_{2} \Delta C_{2}+A_{1} C_{1} \exp \left(\frac{-\delta_{1}}{T}\right)-A_{2} C_{2} \exp \left(\frac{-\delta_{2}}{T}\right)+A_{3} C_{3} \exp \left(\frac{-\delta_{3}}{T}\right), \\
\frac{\partial B_{\varphi}}{\partial t}+M\left(B_{\varphi}\right)=\Delta_{*} B_{\varphi},
\end{array}\right.
$$

where

$$
\Delta q=\frac{\partial^{2} q}{\partial x^{2}}+\frac{1}{r} \frac{\partial}{\partial r}\left(r \frac{\partial q}{\partial r}\right), \Delta q=\frac{\partial^{2} q}{\partial x^{2}}+r \frac{\partial}{\partial r}\left(\frac{1}{r} \frac{\partial q}{\partial r}\right), M(q)=w \frac{\partial q}{\partial x}+u \frac{\partial q}{\partial r}, C_{3}=1-C_{1}-C_{2}
$$

with $C_{1}, C_{2}, C_{3}$ being the mass fractions of thevolatile gas,intermediate product, and the final product, $x=z / r_{0}, w=u_{z} / U_{0}, u=u_{r} / U_{0}, v=r \cdot v_{\varphi}, q$ denotes any of the quantities $\rho ; u ; w ; v ; T ; C_{1} ; C_{2} ; B_{\varphi}$, $P_{2}=D /\left(U_{0} \cdot r_{0}\right)=0.01, P_{1}=\lambda /\left(c_{p} \cdot \rho_{0} \cdot U_{0} \cdot r_{0}\right)=0.05, q_{1}=Q_{1} /\left(c_{p} \cdot T_{0}\right)=5, q_{2}=Q_{2} /\left(c_{p} \cdot T_{0}\right)=1, Q_{1}=1.5 \cdot 10^{6}$ $\left(\mathrm{J} \cdot \mathrm{kg}^{-1}\right), Q_{2}=0.3 \cdot 10^{6}\left(\mathrm{~J} \cdot \mathrm{kg}^{-1}\right)$ are the heat losses in the reactions, $\delta_{k}=E_{k} /\left(R T_{0}\right),\left(\delta_{1}=\delta_{3}=10, \delta_{2}=13\right)$ are the scaled activation energy, $\mathrm{R}=8.314\left(\mathrm{~J} \cdot(\mathrm{mol} \cdot \mathrm{K})^{-1}\right)$ isthe universal gas constant, $E_{1}=E_{3}=2.5 \cdot 10^{5}$ $\left(\mathrm{J} \cdot \mathrm{mol}^{-1}\right), E_{2}=3.2 \cdot 10^{5}\left(\mathrm{~J} \cdot \mathrm{mol}^{-1}\right)$ are the activation energy, $\lambda=0.25\left(\mathrm{~J} \cdot(\mathrm{s} \cdot \mathrm{m} \cdot \mathrm{K})^{-1}\right)$ is the thermal conductivity, $\mathrm{D}=2.5 \times 10^{-4}\left(\mathrm{~m}^{2} \cdot \mathrm{s}^{-1}\right)$ is the molecular diffusivity of the species, $A_{k}=A^{\prime}{ }_{k} \cdot r_{0} / U_{0}$, $\left(A_{1}=5 \cdot 10^{4}, \quad A_{2}=5 \cdot 10^{5}, \quad A_{3}=5 \cdot 10^{4}\right) \quad$ are the scaled pre-exponential factors $A^{\prime}{ }_{k}=1 / \mathrm{s}$, $R e=U_{0} \cdot r_{0} \cdot \rho_{0} / \eta=1000$ is the Reynolds number, $\eta=5 \cdot 10^{-6}\left(\mathrm{~kg} \cdot \mathrm{s} \cdot \mathrm{m}^{-1}\right)$ is the viscosity, $k=1,2,3$.

The equations were made dimensionless by scaling all the lengths to $r_{0}$, the meridian velocity to $U_{0}$, the azimuthal velocity to $V_{0}$, the temperature to $T_{0}$, the pressure to $\rho_{0} \cdot U_{0}^{2}\left(\mathrm{~N} \cdot \mathrm{m}^{-2}\right)$, the current density (between the walls of the combustor and the axially inserted electrode at the combustor inlet)

$$
j_{r}=\frac{-1}{\mu} \frac{\partial B_{\varphi}}{\partial z}, j_{z}=\frac{1}{\mu r} \frac{\partial\left(B r_{\varphi}\right)}{\partial r} \text { to } j_{0}=\frac{I}{2 \pi r_{0}^{2}}=\left[\mathrm{A} \cdot \mathrm{m}^{-2}\right],
$$

the azimuthal induction of the magnetic field to $B_{0}=\mu \cdot I /\left(2 \cdot \pi \cdot r_{0}\right)=\left[\right.$ Tesla $\left.=\mathrm{N} \cdot(\mathrm{Am})^{-1}\right]$, the electromagnetic forces $F_{r}=-B_{\varphi} \cdot j_{z}, F_{z}=B_{\varphi} \cdot j_{r}$ to $F_{0}=j_{0} \cdot B_{0}=\left[\mathrm{N} \cdot \mathrm{m}^{-3}\right]$, where $\mu=4 \pi \cdot 10^{-7}=\left[\mathrm{N} \cdot \mathrm{A}^{-2}\right]$ is the magnetic permeability, $I=0(0.001) 0.01[\mathrm{~A}]$ is the electric current.

The dimensionless radial and axial components of electromagnetic forces

$$
F_{r}=-\frac{B_{\varphi}}{r} \frac{\partial\left(r B_{\varphi}\right)}{\partial r} F_{x}=-B_{\varphi} \frac{\partial B_{\varphi}}{\partial x}
$$

were quantified using the parameter $P_{e}=B_{0} \cdot j_{0} \cdot r d\left(\rho_{0} \cdot U_{0}^{2}\right)$. For the dimensionless pressure $p$ a model for perfect gas is used: $p=\rho T$.

The boundary conditions are the following [7]:

1. $r=0$ (along the axis): $u=v=0, \partial T / \partial r=\partial C_{k} / \partial r=\partial w / \partial r=0, B_{\varphi}=0$,

2. $r=1$ (at the wall): $u=v=0, \partial w / \partial r=\partial C_{k} / \partial r=0, \partial T / \partial r+B_{i}(T-1)=0, B_{\varphi}=B_{0} \cdot\left(1-x / x_{0}\right)$ (consistent with uniform distribution of $j_{r}=$ const),

3. $x=x_{0}=2$ (at the combustor outlet): $u=0, B_{\varphi}=0, \partial T / \partial x=\partial C / \partial x=\partial w / \partial x=\partial v / \partial x=0$,

4. $x=0$ (at the combustor inlet): $u=0, T=1, C_{2}=0$ for $r \in[0,1]$ and $w=1, C_{1}=1, \mathrm{v}=0$ for

$$
r \in\left[0, \mathrm{r}_{1}\right] ; v=4 r \frac{\left(r-r_{1}\right)(1-r)}{\left(1-r_{1}\right)^{2}},
$$

$w=0, C_{1}=0$; for $r>r_{1}$ we have a uniform jet flow at $r<r_{1}$ and rotation at $r>r_{1} ; B_{\varphi}=B_{0} / r$; for $r$ $\in\left[r_{*}, 1\right]\left(j_{z}=0\right)$ and 


$$
B_{\varphi}=\frac{B_{0}}{r}\left(1-\sqrt{1-\frac{r^{2}}{r^{2}}}\right)
$$

for $r \in\left[0, r_{*}\right][9]$. Here

$$
B i=\frac{h r_{0}}{\lambda}=0.1
$$

is the Biot number, $r_{1}=0.75, r_{*}=0.2, h=0.1 \mathrm{~J} \cdot\left(\mathrm{s} \cdot \mathrm{m}^{2} \mathrm{~K}\right)^{-1}$.

\section{Results and discussion}

To solve a discrete $2 \mathrm{D}$ problem with $40 \times 80$ uniform grid points and the time step 0.0008 , the ADI method of Douglas and Rachford [10] was used. For the stationary solution with the maximal error $10^{-7}$, approximately 2000-3000 time steps were used (the final time $t_{f}=0.9 \mathrm{~s}$ ). The distribution of the axial, radial and azimuthal components of velocity, density and temperature was calculated with MATLAB (Table 1). For a 1D reaction-diffusion problem, some results were obtained with the Matlab solver "pdepe".

To assess the electric field effects on the gasification/combustion characteristics of the straw-coal mixtures, the experimental studies include complex time-dependent measurements of the mixture weight loss rate during the gasification stage, of the composition of the released volatiles, of the flame temperature, of the heat output from the device and of the composition of the products along with estimation of the electric field effect on the main characteristics for different compositions of the mixtures at different stages of the mixture thermo-chemical conversion.

Table 1

\section{Maximum and minimum values of the main flame characteristics versus} the electromagnetic parameter $\boldsymbol{P}_{e}$

\begin{tabular}{|c|c|c|c|c|c|c|c|c|}
\hline $\boldsymbol{P}_{\boldsymbol{e}}$ & $\boldsymbol{C}_{\boldsymbol{3}, \min }$ & $\boldsymbol{\rho}_{\min }$ & $\boldsymbol{w}_{\max }$ & $\boldsymbol{u}_{\max }$ & $\boldsymbol{u}_{\min }$ & $\boldsymbol{T}_{\max }$ & $\boldsymbol{T}_{\mathrm{av}}$ & $\boldsymbol{C}_{\mathbf{2}, \max }$ \\
\hline 0 & 0.8022 & 0.033 & 4.58 & 2.61 & 0 & 3.650 & 3.376 & 0.4056 \\
\hline 0.1 & 0.8025 & 0.036 & 4.59 & 2.62 & -0.11 & 3.667 & 3.379 & 0.4062 \\
\hline 0.2 & 0.8029 & 0.039 & 4.60 & 2.63 & -0.25 & 3.693 & 3.384 & 0.4066 \\
\hline 0.5 & 0.8037 & 0.052 & 4.81 & 2.66 & -0.78 & 3.782 & 3.400 & 0.4066 \\
\hline 1.0 & 0.8013 & 0.055 & 5.31 & 2.70 & -1.73 & 3.910 & 3.400 & 0.4049 \\
\hline 2.0 & 0.7850 & 0.048 & 6.67 & 2.74 & -3.43 & 4.192 & 3.333 & 0.4025 \\
\hline
\end{tabular}

From the data summarized in Table 1 it follows that for a mixture of biomass pellets of coal $+20 \%$ straw, the maximum value of the mass fraction of the intermediate product $\left(C_{2, \max }\right)$, the minimum value of the mass fraction of the final product $\left(C_{3, \text { min }}\right)$ and the average temperature $\left(T_{\mathrm{av}}\right)$ increase at $P_{e}<0.5$ and then decrease at $P_{e}>0.5$. A similar situation was observed for the maximum value for $C_{2}$ at outlet $\left(C_{2, \text { end } \max }=1-C_{3, \min }\right)$ : the maximum temperature value $\left(T_{\max }\right)$, the axial velocity $\left(w_{\max }\right)$, the radial velocity $\left(u_{\max }\right)$ and the negative values for $\mathrm{u}\left(u_{\min }\right)$ increased.

The time-dependent variations and the development of the space distribution of the temperature, axial and radial velocity and concentrations $(0<r<1,0<x<2)$ are presented in Fig. 2-5. The mass fractions of the products increase, but the mass fraction of the reactants decreases to zero with time. Similar variations of $C_{1}$ and $C_{2}$ were observed, when the electric field was applied to the flame.

Figs. 5-7 illustrate the electric field effect on the development of the flame velocity profiles of the time-dependent variations of the mass fractions of $C_{1}, C_{2}$ for $P_{e}=0$ and for $P_{e}>0$. The vorticity of the radial velocity increases with increasing the electric field (Fig. 6, 7).

The experimental study of the electric field effect on the processes developing at the cocombustion of straw with crashed coal has shown complex variations of the mixture weight loss rate, composition of the produced volatiles at the inlet of the combustor, produced heat energy per mass of 
burned mixture, products' composition and temperature (Fig. 8, a-f), which depend on the positive bias voltage of the axially inserted electrode and on the mass fraction of straw in the mixture.

a)

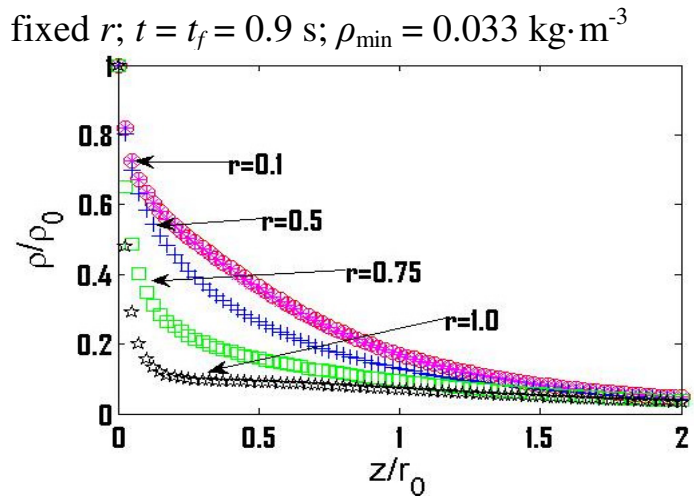

b)

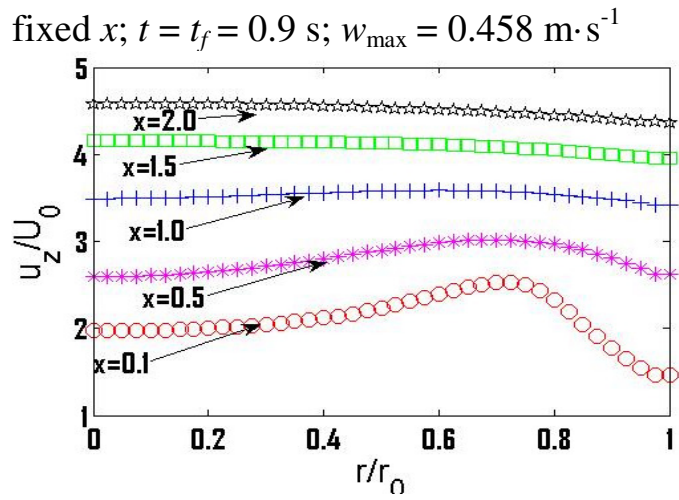

Fig. 2. Development of axial distribution of flow density (a) and radial distribution of velocity (b) at $\boldsymbol{P}_{e}=\mathbf{0}$

fixed $t=t_{f}=0.9 \mathrm{~s} ; T_{\max }=1095 \mathrm{~K} ; T_{0}=300 \mathrm{~K}$

a)

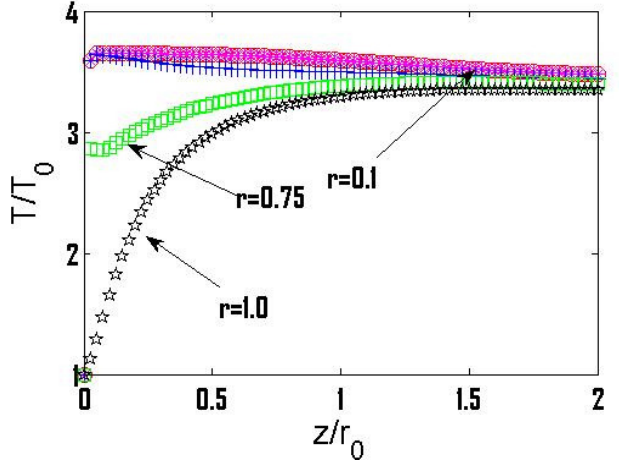

b)

fixed $t=t_{f}=0.9 \mathrm{~s} ; u_{\max }=0.260 \mathrm{~kg} \cdot \mathrm{m}^{-3} ; u_{\min }=0$

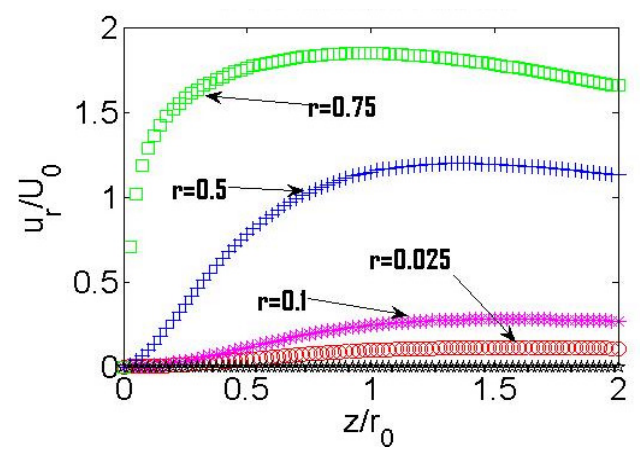

Fig. 3. Development of temperature (a) and velocity axial distribution (b) at $\boldsymbol{P}_{e}=\mathbf{0}$

a)

$$
C_{1}=1, C_{1, \min }=0 \text { for } t>0.0005 \mathrm{~s}
$$

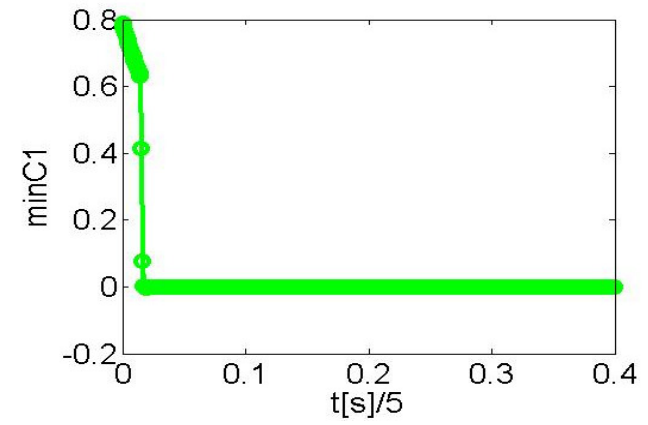

b)

$$
C_{2}=0, \mathrm{C}_{2, \max }=0.4056 \text { for } t>0.5000 \mathrm{~s}
$$

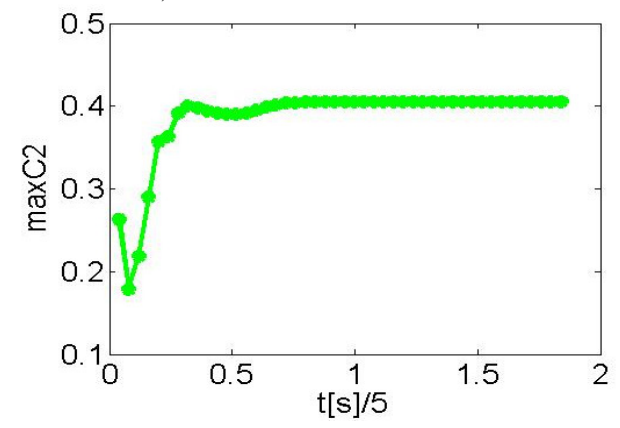

Fig. 4. Time dependent variations of reactants (C1) (a) and products (C2) (b) at combustor inlet

fixed $t=t_{f}=0.9 \mathrm{~s} ; w_{\max }=0.458 \mathrm{~m} / \mathrm{s}, w_{\min }=0$

a)

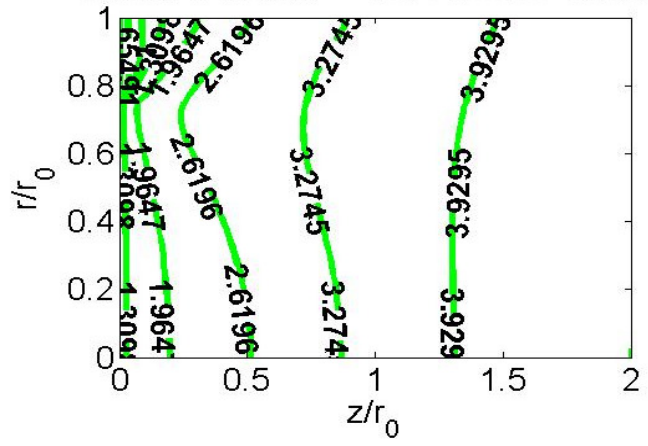

b)

fixed $t=t_{f}=0.9 \mathrm{~s} ; u_{\max }=0.261 \mathrm{~m} / \mathrm{s}, u_{\min }=0$

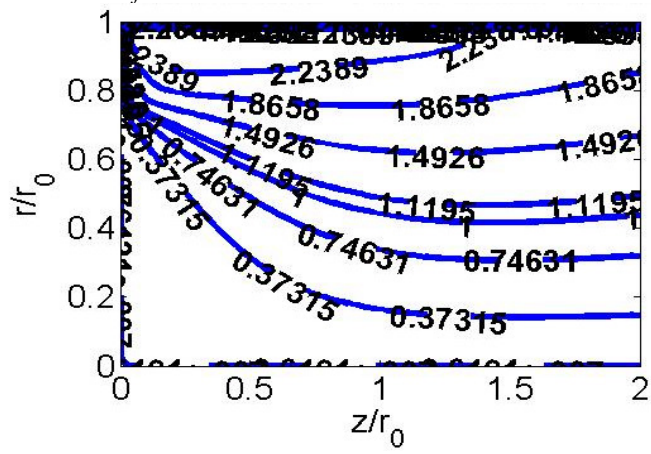

Fig. 5. Development of radial profiles of axial (a) and radial (b) velocity downstream combustor at $\boldsymbol{P}_{e}=0$ 


$$
\text { fixed } t=t_{f}=0.9 \mathrm{~s} ; w_{\max }=0.481 \mathrm{~m} \cdot \mathrm{s}^{-1} ; w_{\min }=0
$$

a)

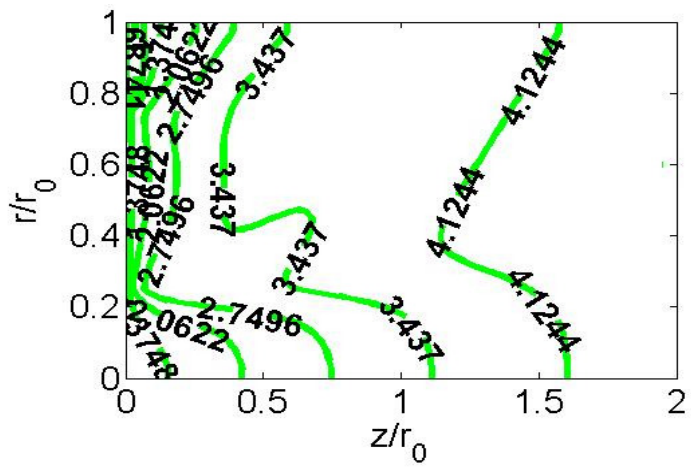

b)

$$
\text { fixed } \begin{gathered}
t=t_{f}=0.9 \mathrm{~s} ; u_{\max }=0.266 \mathrm{~m} \cdot \mathrm{s}^{-1} ; \\
u_{\min }=-0.077 \mathrm{~m} \cdot \mathrm{s}^{-1}
\end{gathered}
$$

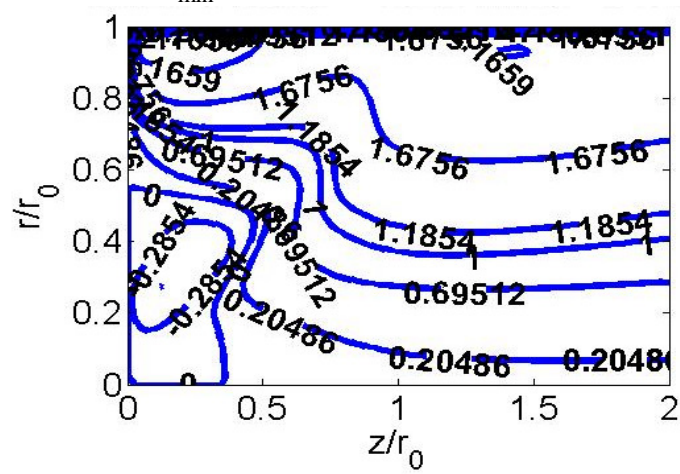

Fig. 6. Electric field effect on development of axial (a) and radial (b) velocity profiles downstream combustor at $\boldsymbol{P}_{e}=0.5$

fixed $t=t_{f}=0.9 \mathrm{~s} ; w_{\max }=0.531 \mathrm{~m} \cdot \mathrm{s}^{-1} ; w_{\min }=0$

a)

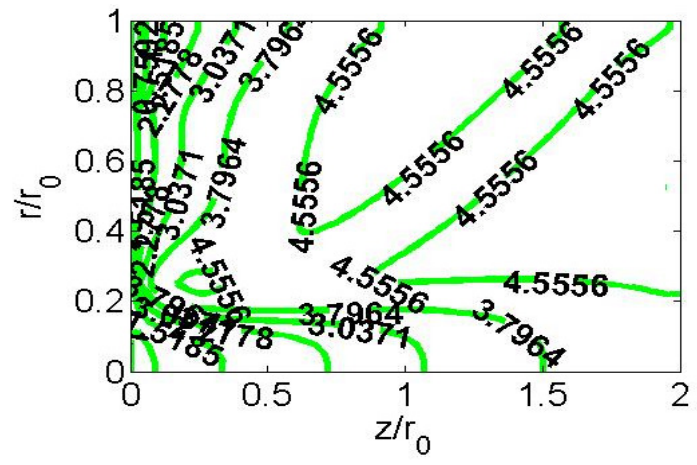

b)

$$
\text { fixed } \begin{gathered}
t=t_{f}=0.9 \mathrm{~s} ; u_{\max }=0.270 \mathrm{~m} \cdot \mathrm{s}^{-1} ; \\
u_{\min }=-0.173 \mathrm{~m} \cdot \mathrm{s}^{-1}
\end{gathered}
$$

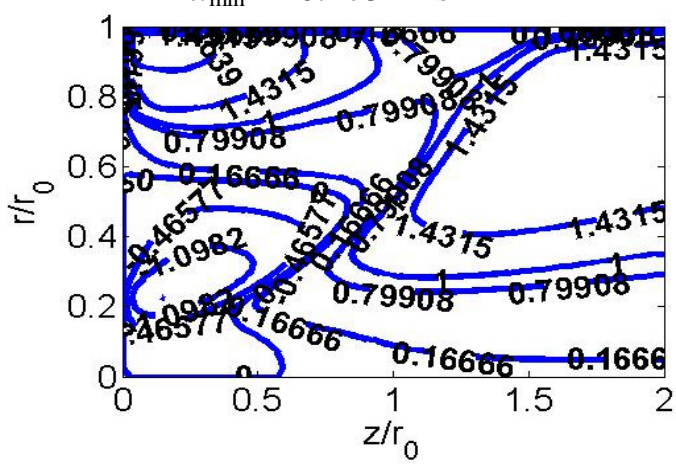

Fig. 7. Electric field effect on development of axial (a) and radial (b) velocity profiles downstream combustor at $\boldsymbol{P}_{e}=\mathbf{1 . 0}$

A decrease of the mixture weight loss rate with the correlating decrease of the mass fraction of the combustible volatiles entering the combustor and of the produced heat energy was observed, when increasing the bias voltage up to $1.2 \mathrm{kV}$, when the field-induced body force promotes the fieldenhanced radial and reverse axial drift motion of the charged flame species $\left(\mathrm{CHO}^{+}, \mathrm{C}_{3} \mathrm{H}_{3}{ }^{+}, \mathrm{CHO}^{+}\right)$ in the field direction [1]. The elastic collisions between the ions and neutral flame species with momentum transfer to the bulk gases enhance the processes of heat/mass transfer (ion wind effect), which led to the radial flame expansion and to the marked decrease of the flame length. The flame temperature decreased below $550 \mathrm{~K}$ restricting thus the thermal decomposition of wheat straw pellets, the formation of combustible volatiles and the transition to self-sustaining combustion of volatiles, which promotes the flame extinction.

The results show that the ion wind induced variations of the flame structure and the main combustion characteristics are influenced by the variations of the straw mass fraction in the mixture, which is responsible for the formation of combustible volatiles and flame ions. The peak values of the average ion current in the space between the electrodes and the average ion density at the flame base 1.4-3.5 $10^{12} \mathrm{~cm}^{-3}$ are detected for a mass fraction of straw in the mixture about 10-20\%. Under such conditions, the electric body force supports the most pronounced flame extinction with the correlating decrease to minimum of the straw-coal mixture weight loss rate, combustible volatiles release, $\mathrm{H}_{2} \mathrm{O}$ absorption at the flame base, heat energy produced in the flame reaction zone and of the $\mathrm{CO}_{2}$ volume fraction in the products (Figs.8-a, b).

The variations of the electric field action on the main flame characteristics were observed when increasing the bias voltage of the electrode above $1.2 \mathrm{kV}$ and when the current-voltage characteristics indicate a gradual transition from the regime of ion current saturation to a discharge with a rapid increase of the current to 6-7 mA and with the correlating increase of the $\mathrm{CO}_{2}$ volume fraction in the products (Fig. 8-e), of the produced heat energy by about 7-8\% (Fig. 8-c) and of the products 
temperature Fig. 8-f). This suggests that increasing the bias voltage results in overlapping of the effects related to the field-enhanced excitation of the flame species at inelastic collisions with free electrons and to the Lorentz force effect on the rate of reactions, which leads to enhanced mixing of the reactants and which is confirmed by the results of the mathematical modelling (Figs. 6, 7). Finally, it should be noted that the main field effect on the flame characteristics was predominately observed during the primary stage of volatiles formation, ignition and combustion and it gradually decreased during the transition to the char conversion stage.
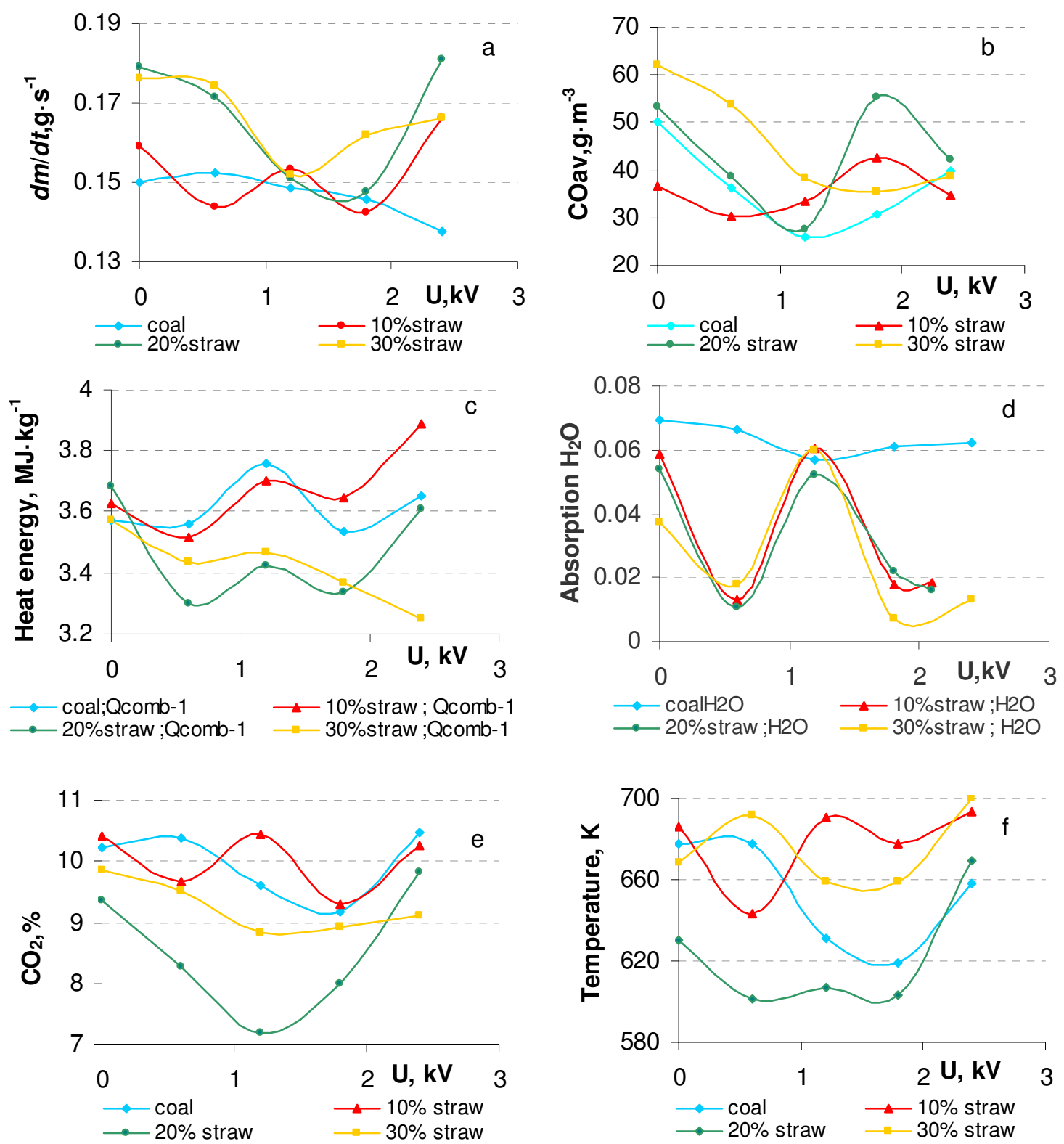

Fig. 8. Electric field-induced variations of weight loss rate (a), density of volatiles entering the combustor (b), produced heat energy in the flame reaction zone (c), $\mathrm{H}_{2} \mathrm{O}$ absorption (d), $\mathrm{CO}_{2}$ volume fraction (e) and products temperature (f)

\section{Conclusions}

1. The results of the numerical simulation have revealed that the formation of the flame reaction zone is influenced by the electric field-induced variations of the flow velocity, temperature and by the mixing of the reactants, when increasing the electric current to $I<0.005 \mathrm{~A}\left(P_{e}<0.5\right)$.

2. The results of the experimental study suggest that the dominant electric field effect on the main gasification/combustion characteristics is determined by the bias voltage of the axially inserted electrode and by the mass load of straw in the mixture advancing so the field-enhanced reverse 
and radial mass transfer with flame extinction at $U<1.2 \mathrm{kV}$ along with the enhanced chemical conversion of the volatiles at $U>1.2 \mathrm{kV}$, with the correlating increase of the produced heat energy, which can be related to overlapping of the effects of the field-enhanced excitation of the flame species at inelastic collisions with free electrons and to the Lorentz force-enhanced mixing of the reactants.

\section{Acknowledgements}

The authors would like to express their gratitude for financial support from the European Regional Funding (project SAM 1.1.1.1./16/A/004).

\section{References}

[1] Lawton J., Weinberg F.J., 1969, Electric Aspects on Combustion, Clarendon Press, Oxford.

[2] Colannino J., 2012, Electrodynamic combustion control, A Clear Sign White Paper, Seattle, pp. 1-11.

[3] Zake M., Turlajs D., Purmalis M., 2000, Electric field control of NOx formation in the flame channel flows, Global Nest: the International Journal, vol.2, no. 1, pp. 99-109.

[4] Xu K.G., 2014, Plasma sheath behavior and ionic wind effect in electric field modified flames, Combustion and Flame, 161, pp.1679-1686.

[5] Barmina I., Purmalis M., Valdmanis R., Zaķe M., 2016, Electrodynamic control of the combustion characteristics and heat energy production, Combustion Science and Technology, vol. 188 (2), 190-206, http://www.tandfonline.com/doi/full/10.1080/00102202.2015.10880 10 ISSN 00102202.

[6] Zhang Y., Wu Y., Yang H., Zhang H., Zhu M., 2013, Effect of high-frequency alternating electric fields on the behavior and nitric oxide emission of laminar non-premixed flames, Fuel, 109, pp. 350-355.

[7] Kalis H., Barmina I., Zake M., Koliskins A., 2016, Mathematical modelling and experimental study of electrodynamic control of swirling flame flows, Engineering for Rural Development, pp. 54-59.

[8] Wesley F., 1980, Table of recommended rate constants for chemical reaction occurring in combustion, National Standard Reference Date System (NSRDS), U.S. Department of Commerce, $115 \mathrm{p}$.

[9] Boyarevitch V.V., Freiberg Y. Zh., Shilova E.I., Shcherbinin E.V., Electro-Vortex Flows, Zinatne Press, Riga (in Russian), 1985, 315 p.

[10] Douglas J., Rachford R., On the numerical solution of heat conduction problems in two and three space variables, Trans. Amer. Math. Soc., vol. 82, 2, 1956, pp. 421-439. 